AperTO - Archivio Istituzionale Open Access dell'Università di Torino

\title{
Low-temperature rapid thermal CVD of nanocrystalline graphene on Cu thin films
}

\section{This is the author's manuscript}

Original Citation:

Availability:

This version is available http://hdl.handle.net/2318/158395

since

Published version:

DOI:10.1002/pssb.201451219

Terms of use:

Open Access

Anyone can freely access the full text of works made available as "Open Access". Works made available under a Creative Commons license can be used according to the terms and conditions of said license. Use of all other works requires consent of the right holder (author or publisher) if not exempted from copyright protection by the applicable law. 


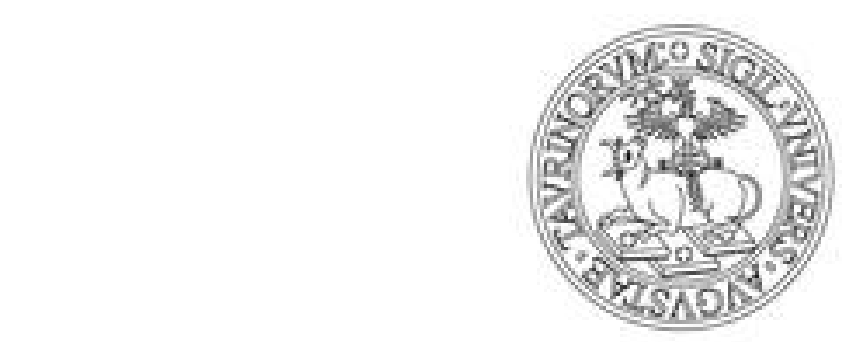

\section{UNIVERSITÀ DEGLI STUDI DI TORINO}

This is an author version of the contribution published on:

Luca Croin,Ettore Vittone,Giampiero Amato

Low-temperature rapid thermal CVD of nanocrystalline graphene on $\mathrm{Cu}$ thin films

PHYSICA STATUS SOLIDI B-BASIC RESEARCH (2014) 251

DOI: $10.1002 / \mathrm{pssb} .201451219$

The definitive version is available at:

http://doi.wiley.com/10.1002/pssb.201451219 


\title{
Low temperature Rapid Thermal CVD of nano-crystalline graphene on Cu thin films
}

\author{
Luca Croin ${ }^{*}, 1,2$, Ettore Vittone ${ }^{3}$ and Giampiero Amato ${ }^{2}$ \\ ${ }^{1}$ Dept. of Applied Science and Technology - DISAT, Politecnico di Torino, Corso Duca degli Abruzzi 24, 10129 Torino, Italy \\ ${ }^{2}$ The Quantum Research Lab, INRiM, Strada delle Cacce 91, 10135 Torino, Italy \\ ${ }^{3}$ Physics Department, University of Turin and NIS Interdepartmental Centre, Via P. Giuria 1, 10125 Torino, Italy
}

Received ZZZ, revised ZZZ, accepted ZZZ

Published online ZZZ (Dates will be provided by the publisher.)

Keywords Rapid Thermal CVD, Graphene, Cu thin film, Raman spectroscopy.

* Corresponding author: e-mail I.croin@inrim.it, Phone: +00 390113919331,

\begin{abstract}
Chemical Vapor Deposition (CVD) of graphene onto $\mathrm{Cu}$ substrates is a very promising approach for large scale production. When dealing with $\mathrm{Cu}$ thin films instead of foils, there are additional problems related to the stability of the film at high temperatures. We show that the film rupture and agglomeration can be prevented by monitoring in-situ dewetting dynamics of the catalytist. We investigated the possibility to perform CVD of graphene onto $\mathrm{Cu}$ films, $200 \mathrm{~nm}$ thick, at low pressure conditions, with ethanol or methane as $\mathrm{C}$ precursors. Same recipes applied on $\mathrm{Cu}$ foils lead to worse results highlighting the important role played by the substrate thickness to achieve a high catalytic activity.

The influence on the deposition quality of parameters such as time, temperature and hydrogen flow is then discussed.
\end{abstract}

1 Introduction Large scale production of graphene is the first step that, once reached with a good reliability, will lead to a large integration of graphene in devices. Chemical Vapor Deposition (CVD) allows the growth of polycrystalline graphene monolayers with grain dimensions that can vary from hundreds of nanometers up to few millimeters. The best results in terms of grains dimension have been obtained on $\mathrm{Cu}$ foils [1] and recently on Pt foils [2] suggesting foil support as a good candidate for large scale production. The main drawback of deposition onto foils is the need for a transferring process that is mainly realised through an intermediate step strengthening the layer by means of a polymer [3].

A promising approach to mitigate this problem is to reduce the substrate thickness and several studies have been carried out centred mainly on $\mathrm{Cu}$ [4-8] and $\mathrm{Ni}$ [8-10] thin films. Moreover, further studies have been reported regarding the relation between crystallinity of the $\mathrm{Ni}$ thin films and the graphene deposition quality [11], while on $\mathrm{Cu}$ thin films some results are given on the influence of the grain orientation ascribing a higher quality to growth onto $<111>$ facets [4,12].

Graphene deposited on films can be transferred with the same methods used for foils, but also other approaches can be followed such as face-to-face transfer [13], rigid transfer [14] or avoiding the use of a polymer and transferring graphene onto silanized $\mathrm{SiO}_{2}$ [15]. Moreover, the dewetting phenomenon [16], which is generally recognized as being a limiting factor for the use of thin films as suitable substrates for graphene deposition, has been exploited to let graphene lay down on the $\mathrm{SiO}_{2}$ substrate beneath the $\mathrm{Cu}$ film [17,18]. All these possibilities suggest a much wider spectrum of applications with respect to graphene grown on foils and envisage new solutions that can lead to cheaper and more effective production processes. In conclusion, the choice of thin films is related to the promising opportunities given by integration in the current microelectronics technology.

Due to dewetting, deposition temperatures lower than the ones used in foils are advisable with thin films. Moreover, lowering temperature could reduce the stress of the sample, a wider range of substrates could be used, and, finally, in an industrial perspective, less power is needed.

Ethanol seems a promising precursor, due to its lower cracking temperature [19], and has been recently used to deposit graphene on $\mathrm{Cu}$ foils at temperatures lower than on methane [20,21]. 
In this paper we present our results in low temperature nano-crystalline graphene growth on thin films by ethanol and methane, investigating different deposition conditions such as hydrogen flow, exposure time and temperature to assess their role in the deposition process.

2 Experimental $\mathrm{Cu}$ thin films used in this work were deposited with an electron beam evaporator onto $\mathrm{SiO}_{2}(285$ $\mathrm{nm}) / \mathrm{Si}$ substrates. Before the deposition, substrates have been cleaned in a class 100 clean room by sonication in acetone and isopropyl alcohol, rinsed in deionized water and dried with nitrogen flow. Depositions were performed in high vacuum at a rate of $0.17 \mathrm{~nm} / \mathrm{s}$. As deposited samples, characterized via Scanning Electron Microscopy (SEM), show an average grain size in the $20-50 \mathrm{~nm}$ range. (Fig. 1A).

To prevent the oxidation of $\mathrm{Cu}$ surface, evaporated samples were rapidly loaded into the Rapid Thermal CVD (RTCVD) system Jipelec JetFirst 100C and pumped down to the $10^{-5}$ mbar range. The RTCVD is a cold-wall heating system and is capable of very fast heating (up to $50{ }^{\circ} \mathrm{C} / \mathrm{s}$ ) by means of an array of 12 halogen lamps; moreover, several gas lines, $\mathrm{N}_{2}, \mathrm{Ar}, \mathrm{H}_{2}$, and ethanol (vapors) are present (a scheme of the system has been provided elsewhere [22]). The temperature control in the RTCVD system is provided by three thermocouples and a pyrometer. We used thermocouples for temperature monitoring during CVD process, and found a new application of the pyrometer to monitor the dewetting phenomena. In fact, as reported in a previous paper [23], at constant temperature the signal from the pyrometer is related to the emissivity $\varepsilon$ of the sample surface. Therefore, being the emissivity of the $\mathrm{Cu}$ thin film rather different from the underlying $\mathrm{SiO}_{2}$ substrate $\left(\varepsilon_{\mathrm{SiO}_{2}}>\varepsilon_{\mathrm{Cu}}\right)$, the time evolution of the infra-red emission from the sample can be attributed to the evolution of the $\mathrm{Cu}$ coverage of the substrate.

Dewetting dynamics is characterized by three typical stages: incubation stage, hole opening and holes propagation; correspondingly, three typical times $t_{0}, t_{1}$ and $t_{2}$ can be extracted [23]. In Table 1 are reported $t_{0}, t_{1}$ values obtained from experiments performed in vacuum $\left(10^{-2} \mathrm{mbar}\right)$ and $\mathrm{H}_{2}$ environment $\left(0.25 \mathrm{mbar}\right.$, in brackets) on $200 \mathrm{~nm} \mathrm{Cu}$ thin films $\left(t_{2}\right.$ is in the 100-300 $s$ range and is not reported). For graphene deposition purposes, the most important time is $t_{0}$, below which the film rupture does not occur and, as evidenced by $t_{0}$ values in brackets, can be increased by the addition of a gas partial pressure. This evidence justifies the choice of a pre-deposition step in which both $\mathrm{H}_{2}$ and $\mathrm{Ar}$ are provided preventing dewetting and at the same time reducing $\mathrm{Cu}$ oxides [24]. Even if film rupture is not reached during incubation stage, grooves at grain boundaries become deeper and deeper till to the film thickness value. This is the major contribution to the increase of film roughness and provokes the puckering of graphene film after transfer [14]. As evidenced in [23], SEM analysis shows an increase of surface roughness on $\mathrm{Cu}$ film during the incubation period with no noticeable variation of the pyrometer signal, if compared to hole opening process which follows.

Table 1 Typical $t_{0}$ and $t_{l}$ times of dewetting dynamics of a $200 \mathrm{~nm} \mathrm{Cu}$ film. Values are relative to experiments executed in vacuum environment $\left(10^{-2} \mathrm{mbar}\right)$ while values in brackets correspond $\mathrm{H}_{2}$ environment $(0.25 \mathrm{mbar})$; hyphen is reported when $t_{0}$ was not measurable. For $800{ }^{\circ} \mathrm{C}$ the value in bold relates to $\mathrm{Ar}(0.25$ mbar $)$ environment.

\begin{tabular}{ccccc}
\hline & $600{ }^{\circ} \mathrm{C}$ & $700{ }^{\circ} \mathrm{C}$ & $800{ }^{\circ} \mathrm{C}$ & $900{ }^{\circ} \mathrm{C}$ \\
\hline$t_{0}$ & $100(980)$ & $12(410)$ & $1\left(14,>10^{3}\right)$ & $-(-)$ \\
$t_{1}$ & $56\left(>10^{3}\right)$ & $23(85)$ & $22(39)$ & $13(25)$ \\
\hline
\end{tabular}

CVD processes were mostly carried out at $700{ }^{\circ} \mathrm{C}$ (except from one at $800{ }^{\circ} \mathrm{C}$ ) following a typical 2 -step scheme composed by annealing and deposition. The process temperature is reached in $35 \mathrm{~s}$ following a $20^{\circ} \mathrm{C} / \mathrm{s} \mathrm{ramp}$, then the annealing step lasts 6 min and is carried out in a fixed atmosphere of $\mathrm{Ar}$ and $\mathrm{H}_{2}\left(\mathrm{Ar}: \mathrm{H}_{2}\right.$ 100:10). Gas composition during deposition step has been changed maintaining the ethanol partial pressure fixed and varying the hydrogen flow (stable gas regimes are reached within $30 \mathrm{~s}$ for all the processes). During cooling a flux of $100 \mathrm{sccm}$ of Ar is maintained. The whole CVD process lasts less that 15 minutes.

Morphological characterization of samples has been performed with a FEI Inspect F Scanning Electron Microscope. Raman spectroscopy has been performed with a Renishaw InVia Raman spectrometer, equipped with a $442 \mathrm{~nm}$ laser (Kimmon IK Series He-Cd Laser) interfaced to a microscope with a 50x objective. With the chosen laser wavelength, it is possible to characterize directly graphene on the catalyst reducing the luminescence emission [25] arising from $\mathrm{Cu}$. Raman spectra were analysed first by subtracting a parabolic baseline, and then peaks were fitted by Lorentzian line shapes. Raman maps were collected over a 2x2 mm area by means of a Renishaw inVia Reflex equipped with a $514.5 \mathrm{~nm}$ laser. 
3 Results In Figure 1 a comparison between typical as-evaporated $\mathrm{Cu}$ and after the CVD process, is shown. It is possible to notice that, according to [26] and [27], the average grain size of Cu polycrystalline thin film increases from nanometer up to micrometer size.

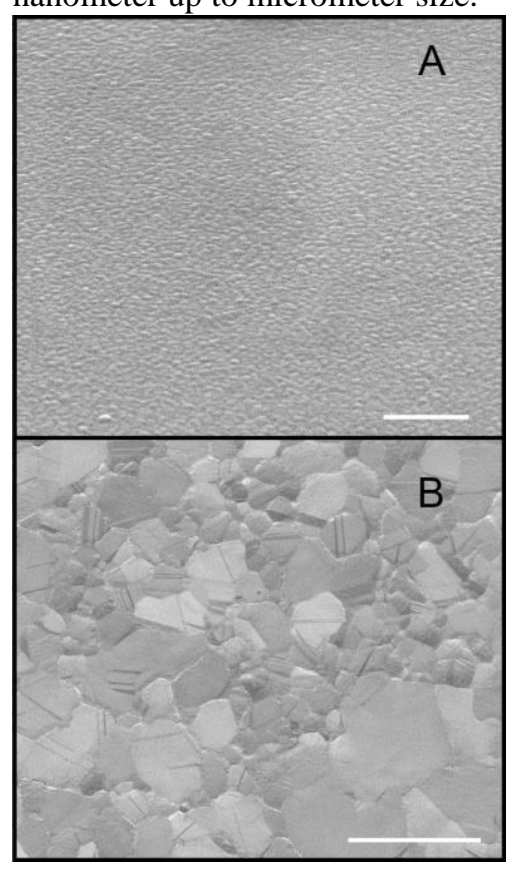

Figure 1 SEM micrographs of: a) an as-evaporated $\mathrm{Cu}$ film (scale bar $500 \mathrm{~nm}$ ), b) sample after the CVD process \#5 (scale bar $5 \mu \mathrm{m}$ ).

Firstly, we applied the CVD process described above (providing only ethanol during graphene deposition) on a $\mathrm{Cu}$ thin film and a foil. In Fig. 2, representative Raman spectra are displayed. The spectrum obtained for the $\mathrm{Cu}$ foil, concordant with the one reported in [20], show $\mathrm{I}_{\mathrm{D}} / \mathrm{I}_{\mathrm{G}} \sim 1.4$ and $\mathrm{I}_{2 \mathrm{D}} / \mathrm{I}_{\mathrm{G}} \sim 0.3$ and can be ascribed to nano-crystalline/amorphous graphene [28] while features of monolayer graphene disappear. On the other hand, a completely different behaviour is observed in thin film where an intense 2D peak, and a narrower G peak are present. An intense D peak indicating that small crystallites are obtained is also present. Furthermore, the intensity of D+D' peak at $2950 \mathrm{~cm}^{-1}$ [29] is not negligible. The same CVD protocol has also been applied to a thin film changing the precursor to methane $\left(\mathrm{CH}_{4}\right.$ flux $\left.=10 \mathrm{sccm}\right)$. Interestingly similar results to ethanol-CVD (cf. Fig. 2) are obtained, indicating that in this range of temperatures the catalytic activity of the film is equally efficient.

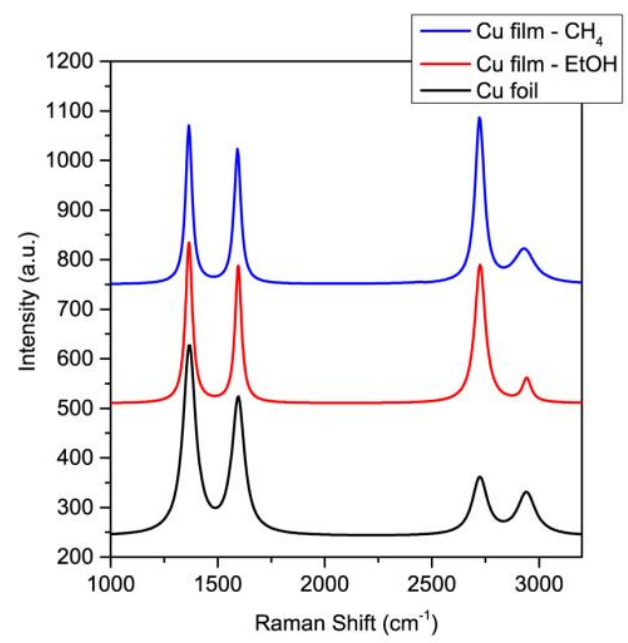

Figure 2 Raman spectra of graphene on $\mathrm{Cu}$ foil and thin film (with ethanol and methane as precursors) after CVD process. 
A possible explanation of the different behaviour noticed in $\mathrm{Cu}$ films instead of foils can be envisaged, assuming a different mobility of atoms in the two substrates. Thin films dewetting, in fact, is related to the unbalance of surface and grain boundary energies [26]. The last is lower in thin films rather than in foils: this enhances the mobility of surface atoms giving rise to dewetting and, probably, enhancing the catalytic activity of the film, e.g. by an augmented hydrogenation [4].

The effect of addition of $\mathrm{H}_{2}$ (in the range 0-20 sccm) during the ethanol deposition had then been studied. The choice of such relatively narrow range of $\mathrm{H}_{2}$ flux is imposed by the need to keep the deposition pressure almost constant, in order to have comparable results. It has to be noticed, in fact, that EtOH flux cannot be controlled in our assembly. In Table 2 a summary of these CVD processes is presented. Pressure measurements, as collected with a Pfeiffer FullRange Gauge, were stable along the whole CVD.

Intensities were obtained from the typical G,D and 2D peaks by fitting data with a Lorentzian shape line. Data in fig. 3 are average values obtained by several spectra collected. The D/G and 2D/G peak ratios are summarized. The error bars represent the dispersion of such values throughout the sample surface.

Table 2 Summary of CVD processes. Temperature was fixed at $700{ }^{\circ} \mathrm{C}$ during both annealing and deposition step. Annealing has been carried out under a flow of a mixture of $\mathrm{Ar}: \mathrm{H}_{2}$ 100:10; the ethanol partial pressure is fixed at 0.84 mbar. Data from Raman spectra are average values obtained by multiple acquisitions throughout the sample.

\begin{tabular}{ccccccccc}
\hline Process ID & $\begin{array}{c}\mathrm{H}_{2} \text { flow } \\
(\mathrm{sccm})\end{array}$ & $\begin{array}{c}\text { Pressure } \\
(\mathrm{mbar})\end{array}$ & $\begin{array}{c}\mathrm{D} \text { pos } \\
\left(\mathrm{cm}^{-1}\right)\end{array}$ & $\begin{array}{c}\text { D FWHM } \\
\left(\mathrm{cm}^{-1}\right)\end{array}$ & $\begin{array}{c}\text { G pos } \\
\left(\mathrm{cm}^{-1}\right)\end{array}$ & $\begin{array}{c}\mathrm{G}_{\text {FWHM }} \\
\left(\mathrm{cm}^{-1}\right)\end{array}$ & $\begin{array}{c}\text { 2D pos } \\
\left(\mathrm{cm}^{-1}\right)\end{array}$ & $\begin{array}{c}\text { 2D FWHM } \\
\left(\mathrm{cm}^{-1}\right)\end{array}$ \\
\hline$\# 1$ & 0 & 0.84 & 1362.5 & 40 & 1592 & 34 & 2726 & 60 \\
$\# 2$ & 5 & 0.95 & 1367 & 35 & 1598 & 33 & 2731 & 57 \\
$\# 3$ & 10 & 1.01 & 1365 & 45 & 1594 & 36 & 2724 & 63 \\
$\# 4$ & 15 & 1.09 & 1361.5 & 39 & 1591 & 36.5 & 2719 & 54 \\
$\# 5$ & 20 & 1.15 & 1362 & 37.5 & 1590 & 37 & 2719 & 59 \\
\hline
\end{tabular}

In the $\mathrm{I}_{\mathrm{D}} / \mathrm{I}_{\mathrm{G}}$ diagram (Fig. 3A), it is evident that, through the different processes, the defect peak is never negligible and its relative intensity is higher than 1 . On the other hand, the $\mathrm{I}_{2 \mathrm{D}} / \mathrm{I}_{\mathrm{G}}$ diagram (Fig. 3B) shows a dependence on the $\mathrm{H}_{2}$ flow. In Table 2, the mean position for each peak and its average FWHM along the different processes is presented: there are no particular trends to be underlined apart from a blueshift of $\mathrm{G}$ and $2 \mathrm{D}$ peaks of sample \#2. Figure $3 \mathrm{C}$ shows a representative spectrum from process \#5, in particular in the inset the good agreement between data and the fitting curve chosen as a single Lorentzian, is displayed (442 nm laser has been used). Figure 3D shows a Raman map of $\mathrm{I}_{2 \mathrm{D}} / \mathrm{I}_{\mathrm{G}}$ ratio obtained over a $2 \times 2 \mathrm{~mm}$ area of sample \#5 transferred onto $\mathrm{SiO}_{2}(285 \mathrm{~nm}) / \mathrm{Si}$ substrate. According to Costa et al. [25] the 2D peak intensity compared to $\mathrm{G}$ peak is more intense when investigated with green light, this supports the hypothesis of a monolayer deposition. Moreover, from Raman mapping we can conclude that deposition is fairly uniform, and disuniformities (i.e. grain boundaries) are smaller than the mapping resolution $(140 \mu \mathrm{m})$ of the microscope. 

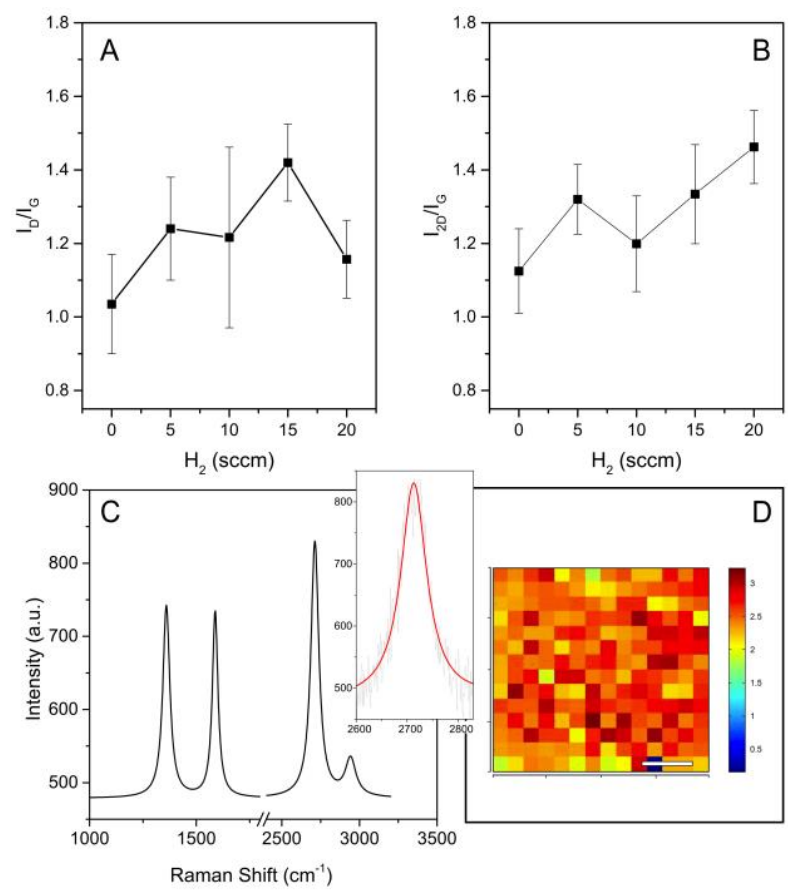

Figure 3 Diagrams related to the processes \#1-\#5: A) $\mathrm{I}_{\mathrm{D}} / \mathrm{I}_{\mathrm{G}}$ ratio, B) $\mathrm{I}_{2 \mathrm{D}} / \mathrm{I}_{\mathrm{G}}$ ratio, C) Raman spectrum of sample \#5 (inset: 2D peak and Lorentzian fit), D) Raman map of $\mathrm{I}_{2 \mathrm{D}} / \mathrm{I}_{\mathrm{G}}$ ratio (sample \#5 transferred) acquired with $514.5 \mathrm{~nm}$ laser (scale bar $0.5 \mathrm{~mm}$ ).

A more detailed analysis of processes \#1-\#5 can be conducted focussing on 2D peak position: ref. [25] gives an empirical formula for the position of the peak for a monolayer graphene on $\mathrm{Cu}$ :

$$
\omega\left(\mathrm{cm}^{-1}\right)=87 \cdot E_{\text {laser }}(\mathrm{eV})+2480
$$

that in our case gives an expected position of $2724 \mathrm{~cm}^{-1}$. This consideration supports the hypothesis of the presence of monolayer, in fact, with an increased number of layers a blueshift should be found. On the other hand, for a monolayer, a $\mathrm{FWHM}_{2 \mathrm{D}} \simeq 28 \pm 5 \mathrm{~cm}^{-1}$ is expected [25]. Our measurements yield a much larger value of $\mathrm{FWHM}_{2 \mathrm{D}}$ that, according to Ferreira et al. [30], is indicative of disorder, in other words, of small grains. For our samples, the estimated grain dimension (extrapolated from [30]) is below $10 \mathrm{~nm}$. To check this evaluation we focused on the $\mathrm{I}_{\mathrm{D}}$ peak. It is indeed well known that from the $\mathrm{I}_{\mathrm{D}} / \mathrm{I}_{\mathrm{G}}$ ratio and the laser wavelength $\lambda$ it is possible to estimate the grain dimension $L_{D}$ [31] as:

$$
L_{D}=2.4 \cdot 10^{-10} \lambda_{\text {laser }}^{4}\left({ }^{I_{D}} / I_{G}\right)^{-1}
$$

$\mathrm{L}_{\mathrm{D}}$ calculation gives for our processes dimensions ranging from 6.5 to $8.9 \mathrm{~nm}$ confirming the previous hypothesis.

Another studied parameter is the deposition time. In Figure 4 the representative spectra of three samples treated with process \#5 are shown with the deposition time as the varying parameter. 


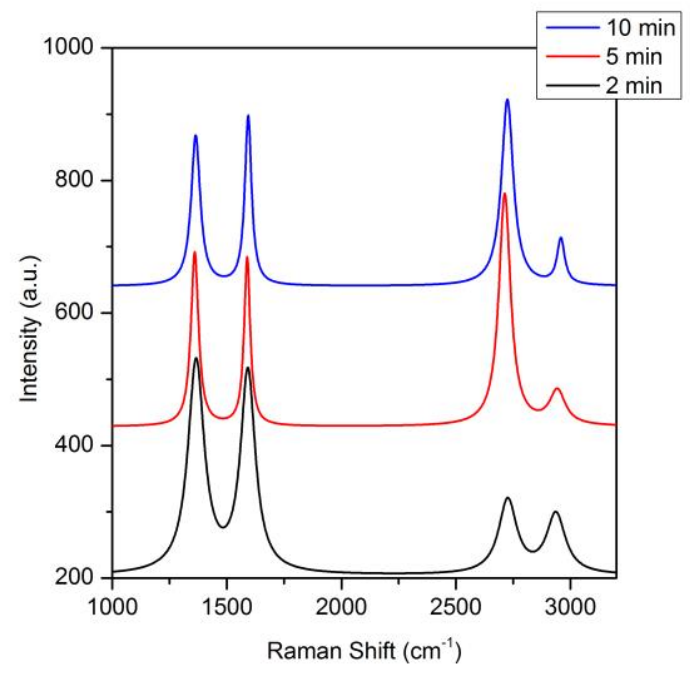

Figure 4 Raman spectra of graphene deposition on $\mathrm{Cu}$ thin film after process \#5 (2 - 5 - $10 \mathrm{~min}$ ).

Basing onto the analysis of the $\mathrm{I}_{2 \mathrm{D}} / \mathrm{I}_{\mathrm{G}}$ ratio, one can conclude that the 2 minutes process yields graphene with poorer quality in terms of crystallinity showing a spectrum similar to the one observed on $\mathrm{Cu}$ foils. The comparison between 5 and 10 minutes is less straightforward: $\mathrm{I}_{2 \mathrm{D}} / \mathrm{I}_{\mathrm{G}}$ is higher in the 5 min process but on the other hand the defect peak is less intense with respect to $\mathrm{G}$ peak in the 10 min process. It is useful to carry on an analysis, as previously done for the $\mathrm{H}_{2}$ flux dependence, investigating peak widths. A more detailed analysis can be carried out by considering the FWHM of the peaks: in particular the $\mathrm{FWHM}_{\mathrm{D}}$ for the 5 min process is $37.5 \mathrm{~cm}^{-1}, 47 \mathrm{~cm}^{-1}$ for the 10 min process while for $\mathrm{FWHM}_{2 \mathrm{D}}$ is $59 \mathrm{~cm}^{-1}$ and $68 \mathrm{~cm}^{-1}$, respectively. Again referring to Ferreira et al. [30] the FWHM is a measure of disorder, therefore at the fixed $\left(700^{\circ} \mathrm{C}\right)$ temperature and hydrogen flux $(20 \mathrm{sccm})$, we can conclude the best graphene quality is obtained with the 5 minutes deposition process.

Finally, we have performed a further analysis by raising the process temperature to $800{ }^{\circ} \mathrm{C}$ with fixed time $(5 \mathrm{~min})$ and $\mathrm{H}_{2}$ flow $(20 \mathrm{sccm})$. In Fig. 5, the comparison of two representative Raman spectra at 700 and $800{ }^{\circ} \mathrm{C}$ (process \#5) is presented.

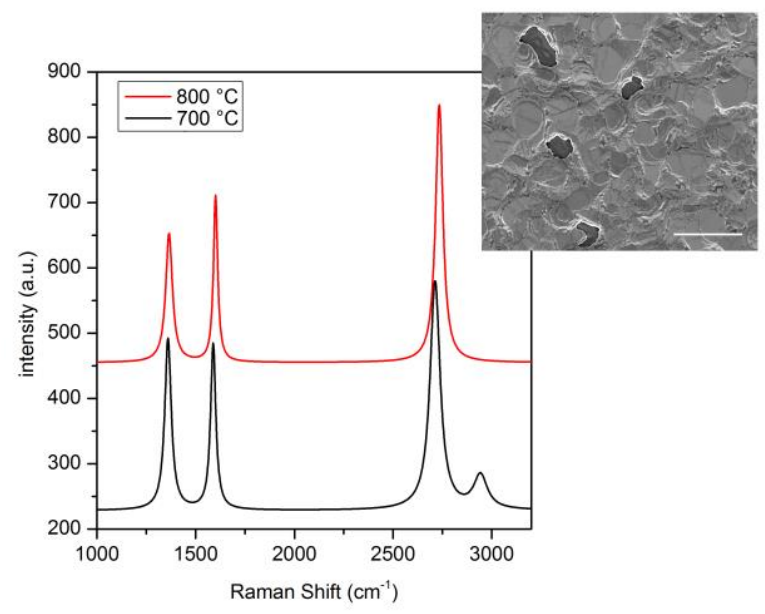

Figure 5 Raman spectra of graphene on $\mathrm{Cu}$ thin film after process $\# 5$ carried out at 700 and $800{ }^{\circ} \mathrm{C}$ (at $800{ }^{\circ} \mathrm{C}$ the $\mathrm{D}+\mathrm{D}$ ' peak intensity was comparable to the detector noise and could not be fitted). Inset: SEM micrograph of the sample at $800{ }^{\circ} \mathrm{C}$ (scale bar $5 \mu \mathrm{m}$ ), showing the early stage of dewetting.

It has been already reported with other substrates or precursors $[20,21,32]$ that increasing temperature the quality of deposition increases. This happens also for our samples, and is confirmed by both the $\mathrm{I}_{2 \mathrm{D}} / \mathrm{I}_{\mathrm{G}}$ ratio and $\mathrm{FWHM}_{2 \mathrm{D}}$. Moreo- 
ver, the small shift that can be observed in 2D peak is again related to graphene domains dimension, in fact when domains are less to $10 \mathrm{~nm}$ a redshift is expected [30].

However, the higher temperature is also responsible of the unwanted first stages of dewetting that consists in the appearance of some holes.

Interestingly, if one performs Raman spectroscopy in holes (on bare $\mathrm{SiO}_{2}$ ) no signatures of graphene are observed in the spectra (Fig. 5). This observation is apparently in contrast with what observed by Ismach et al [17], where graphene deposited on $\mathrm{Cu}$ is then laid on $\mathrm{SiO}_{2}$ when $\mathrm{Cu}$ dewets. Our observation can be explained by following the evolution of the pyrometer signal [23] of the process performed at $800{ }^{\circ} \mathrm{C}$ (Fig. 6). Due to the higher temperature, holes just open during the annealing step and pyrometer signal rises; during deposition step, the graphene, which is deposited on $\mathrm{Cu}$ only, acts as a dewetting limiter. In fact the pyrometer signal during deposition keeps constant. This suggests to reduce the duration of the annealing step to preserve the $\mathrm{Cu}$ film integrity.

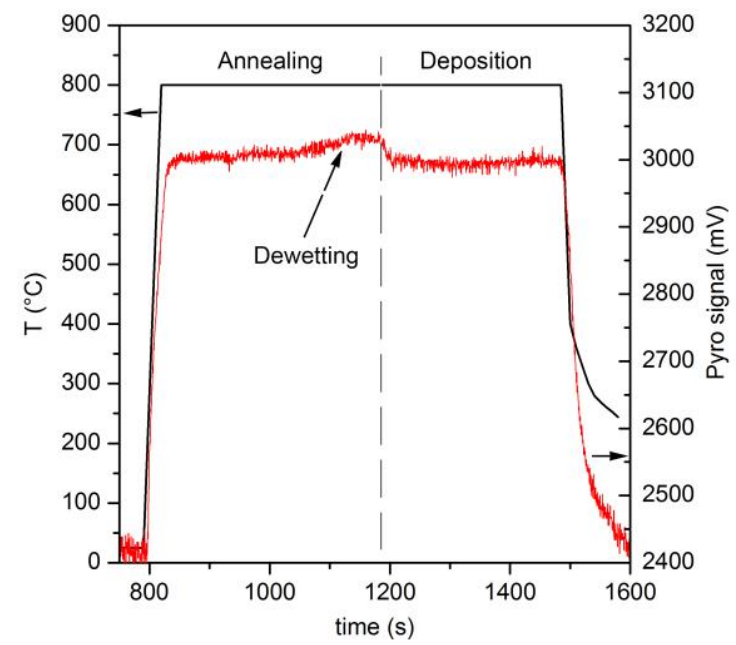

Figure 6 Diagram showing pyrometer signal (red line) and temperature profile (black line) during the process executed at $800{ }^{\circ} \mathrm{C}$. At the end of deposition step pyrometer signal increases showing the early stage of dewetting but during deposition step signal is constant indicating that surface coverage is mantained.

4 Conclusions In conclusion we have reported low temperature growth of nano-crystalline graphene from ethanol onto $\mathrm{Cu}$ thin films. In particular, fixing process temperature at $700{ }^{\circ} \mathrm{C}$, graphene deposition obtained over the entire surface of the $\mathrm{Cu}$ thin film shows a better quality compared to the corresponding graphene deposited on $\mathrm{Cu}$ foil independently if ethanol or methane are used.

The graphene quality seems to be marginally influenced by the $\mathrm{H}_{2}$ flux, at least in the range $(0-20 \mathrm{sccm})$ here chosen, whereas it is evident that deposition time plays a non-negligible role.

Finally, the increase of process temperature cannot be considered as a general way to achieve optimal deposition conditions. In fact, our results evidence that the improvement of the graphene quality in terms of $\mathrm{I}_{2 \mathrm{D}} / \mathrm{I}_{\mathrm{G}}$ and $\mathrm{I}_{\mathrm{D}} / \mathrm{I}_{\mathrm{G}}$ ratios is achieved at expenses of the thin film integrity, which undergoes the dewetting phenomenon. Further studies to optimize the duration of both the annealing and the deposition steps are then needed.

Acknowledgements Authors are indebted with Dr. A Damin (Raman Laboratory, NIS Centre of Excellence, University of Turin) for Raman spectroscopic investigations and useful discussions. This work was partially supported by "Linea 1A ORTO11RRT5" projects of the University of Torino, funded by "Compagnia di San Paolo". Authors wish to thank also Prof. F. Pirri, Prof. F. Giorgis and Dr. A. Virga (Dept. of Applied Science and Technology, Politecnico di Torino) for Raman mapping. 


\section{References}

[1] X. Li, W. Cai, J. An, S. Kim, J. Nah, D. Yang, R. Piner, A. Velamakanni, I. Jung, E. Tutuc, S. K. Banerjee, L. Colombo, and R. S. Ruoff, Science 324, 1312 (2009).

[2] T. Ma, W. Ren, X. Zhang, Z. Liu, Y. Gao, L.-C. Yin, X.-L. Ma, F. Ding, and H.-M. Cheng, Proc. Natl. Acad. Sci. 110, 20386 (2013).

[3] A. Reina, X. Jia, J. Ho, D. Nezich, H. Son, V. Bulovic, M. S. Dresselhaus, and J. Kong, Nano Lett. 9, 30 (2009).

[4] L. Tao, J. Lee, H. Chou, M. Holt, R. S. Ruoff, and D. Akinwande, ACS Nano 6, 2319 (2012).

[5] M. P. Levendorf, C. S. Ruiz-Vargas, S. Garg, and J. Park, Nano Lett. 9, 4479 (2009).

[6] C.-Y. Su, A.-Y. Lu, C.-Y. Wu, Y.-T. Li, K.-K. Liu, W. Zhang, S.-Y. Lin, Z.-Y. Juang, Y.-L. Zhong, F.-R. Chen, and L.-J. Li, Nano Lett. 11, 3612 (2011).

[7] Y.-H. Lee and J.-H. Lee, Appl. Phys. Lett. 96, 083101 (2010).

[8] Y. Lee, S. Bae, H. Jang, S. Jang, S.-E. Zhu, S. H. Sim, Y. I. Song, B. H. Hong, and J.-H. Ahn, Nano Lett. 10, 490 (2010).

[9] W. Liu, C.-H. Chung, C.-Q. Miao, Y.-J. Wang, B.-Y. Li, L.-Y. Ruan, K. Patel, Y.-J. Park, J. Woo, and Y.-H. Xie, Thin Solid Films 518, S128 (2010).

[10]R. S. Weatherup, B. Dlubak, and S. Hofmann, ACS Nano 6, 9996 (2012).

[11]S. Thiele, A. Reina, P. H, J. Kedzierski, P. Wyatt, P.-L. Hsu, C. Keast, J. Schaefer, and J. Kong, Nanotechnology 21, 015601(2010).

[12]M. Ishihara, Y. Koga, J. Kim, K. Tsugawa, and M. Hasegawa, Mater. Lett. 65, 2864 (2011).

[13]L. Gao, G.-X. Ni, Y. Liu, B. Liu, A. H. Castro Neto, and K. P. Loh, Nature 505, 190 (2013).

[14]G. Amato, E. Simonetto, L. Croin, and E. Vittone, in: Proceedings of 2013 MRS Fall Meeting on Large-Area Graphene and Other 2D-Layered Materials - Synthesis, Properties and Applications, Boston, USA, 2013, DOI:10.1557/opl.2014.506.

[15]R. Bajpai, S. Roy, L. Jain, N. Kulshrestha, K. S. Hazra, and D. S. Misra, Nanotechnology 22, 225606 (2011).

[16]C. V. Thompson, Annu. Rev. Mater. Res. 42, 399 (2012).

[17]A. Ismach, C. Druzgalski, S. Penwell, A. Schwartzberg, M. Zheng, A. Javey, J. Bokor, and Y. Zhang, Nano Lett. 10, 1542 (2010).

[18]T. Kaplas, D. Sharma, and Y. Svirko, Carbon 50, 1503 (2012).

[19]S. Duan and S. Senkan, Ind. Eng. Chem. Res. 44, 6381 (2005).

[20]P. Zhao, A. Kumamoto, S. Kim, X. Chen, B. Hou, S. Chiashi, E. Einarsson, Y. Ikuhara, and S. Maruyama, J. Phys. Chem. C 117, 10755 (2013).

[21]A. Guermoune, T. Chari, F. Popescu, S. S. Sabri, J. Guillemette, H. S. Skulason, T. Szkopek, and M. Siaj, Carbon 49, 4204 (2011).

[22]M. Piazzi, L. Croin, E. Vittone, and G. Amato, SpringerPlus 1, 52 (2012).

[23]L. Croin, E. Vittone, and G. Amato, "In situ control of dewetting of $\mathrm{Cu}$ thin films in graphene chemical vapor deposition" Thin Solid Films (accepted).

[24]C. Mattevi, H. Kim, and M. Chhowalla, J. Mater. Chem. 21, 3324 (2011).

[25]S. D. Costa, A. Righi, C. Fantini, Y. Hao, C. Magnuson, L. Colombo, R. S. Ruoff, and M. A. Pimenta, Solid State Commun. 152, 1317 (2012).

[26]C. V. Thompson, Annu. Rev. Mater. Sci. 30, 159 (2000).

[27]S. Simões, R. Calinas, M. T. Vieira, M. F. Vieira, and P. J. Ferreira, Nanotechnology 21, 145701 (2010).

[28]A. Turchanin, D. Weber, M. Büenfeld, C. Kisielowski, M. V. Fistul, K. B. Efetov, T. Weimann, R. Stosch, J. Mayer, and A. Gölzhäuser, ACS Nano 5, 3896 (2011).

[29]A. C. Ferrari and D. M. Basko, Nat. Nanotechnol. 8, 235 (2013).

[30]E. H. Martins Ferreira, M. V. O. Moutinho, F. Stavale, M. M. Lucchese, R. B. Capaz, C. A. Achete, and A. Jorio, Phys. Rev. B 82, 125429 (2010).

[31]M. A. Pimenta, G. Dresselhaus, M.S. Dresselhaus, L. G. Cançado, A. Jorio, and R. Saito, Phys. Chem. Chem. Phys. 9, 1276 (2007).

[32]I. Vlassiouk, S. Smirnov, M. Regmi, S. P. Surwade, N. Srivastava, R. Feenstra, G. Eres, C. Parish, N. Lavrik, P. Datskos, S. Dai, and P. Fulvio, J. Phys. Chem. C 117, 18919 (2013). 